\title{
SKIN IMPEDANCE IMPLICATIONS OF TENS FUNCTION AND THE DEVELOPMENT OF AN IMPROVED STIMULATION WAVEFORM
}

\author{
David C. Johnson, MSEE, BEE \\ Daniel W. Repperger, Ph.D, P.E. \\ Armstrong Laboratory, Building 441, Wright-Patterson AFB OH, 45433
}

\begin{abstract}
A modified Transcutaneous Electric Nerve Stimulator (IENS) stimulation waveform is being used in an experimental prototype unit. The waveform varies from those generated in traditional TENS in shape and spectral content. A pilot study of skin impedance using TENS electrodes was performed. Impedance characteristic Bode plots were made of subjects with prepared and unprepared skin. Spectral magnitudes were then compared to the spectral energies of a traditional TENS device stimulation waveform and the prototype waveform. An effective power transfer ratio was calculated of the two waveforms and the prototype waveform showed a $80 \%$ effective power transfer increase over the traditional waveform. Insight has also been gained into how DC resistance measurements can be performed after epidermal electrical excitation.
\end{abstract}

\section{INTRODUCTION}

A pilot study was conducted to quantify resistive and capacitive characteristics of skin as seen in TENS applications. This was done in two parts, resistance measurements, and complex impedance measurements. Twenty subjects participated, with 10 having prepared skin and 10 having unprepared skin. Standard TENS electrodes were used, placed 5 inches apart on the inside of the forearm. Skin preparation of the prepared group consisted of cleaning the surface with the mild application of an alcohol-pad over a 2 " $\times 2$ " area for no more than 5 seconds. Subjects were $25-40$ year old office workers. Resistance measurements were made with a standard digital multimeter. Impedance magnitude and phase measurements were made with a typical voltage divider arrangement over distributed frequencies across the two electrodes by observation of ascilloscope traces.

\section{IMPEDANCE MEASUREMENTS}

Skin resistance of unprepared subjects typically varied between 120 and $650 \mathrm{KOhms}$. The higher range was associated with darker skin tone and drier complexion. After mild preparation, resistance decreased to between 20 and 60 KOhms. Average skin resistance dropped by $88 \%$ with mild abrasion (i.e. non-discomforting to the point of shallow redness). Subjects having darker skin tones and higher levels of keratin had prepared and unprepared resistances of 130 and $900 \mathrm{KOhms}$, respectively. Here, the average drop in resistance was $80 \%$. African-American subjects tested in the same manner had even greater resistances with average values of prepared and unprepared resistances of 210 and 10,000 KOhms, respectively. Resistance decrease for these was $98 \%$. Overall, preparation consistently decreased skin resistance
$80 \%$ to $98 \%$.

A facet of internal capacitance is the capability of holding charge. DC meastrements of electrode resistance were taken after the frequency information was collected. It was observed that by switching the polarity of the multimeter probes, the measured resistance changed, indicating that an internal charge was present. This may cause errors in resistance measurements. Resonant roltages existed across the electrodes for several minutes after testing, even when externally shorted. This charge affects resistance measurements according to the schematic in Pigure 1.

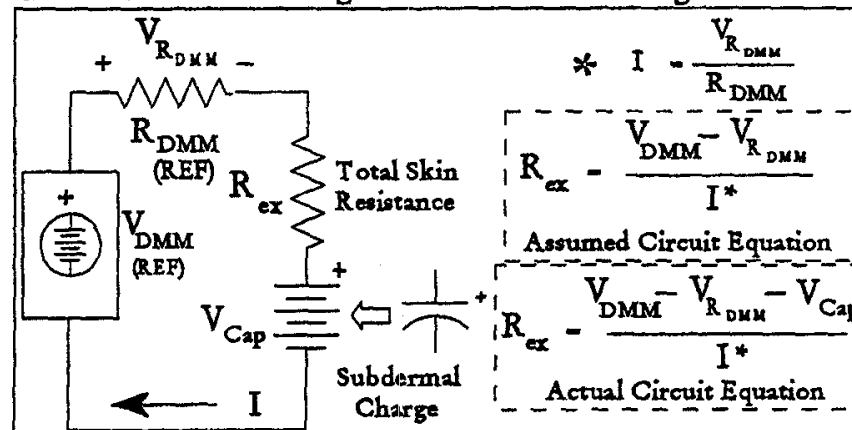

Bgure 1: Multimeter Equivalent Circuit on Skin and Equations

The multimeter measures current across a reference resistor after a reference voltage is applied. A charge on the tissue is analogous to adding a battery in series with the skin. The battery either adds or subtracts current from the reference current. Any DC voltage across electrodes may accrue charge and lead to errors in resistance measurement. This effect was seen in every subject after DC voltage exposure altered resistance measurements by $10 \%-15 \%$ consistently.

Magnitude and phase voltage measurements were collected and are plotted in Figure 2.

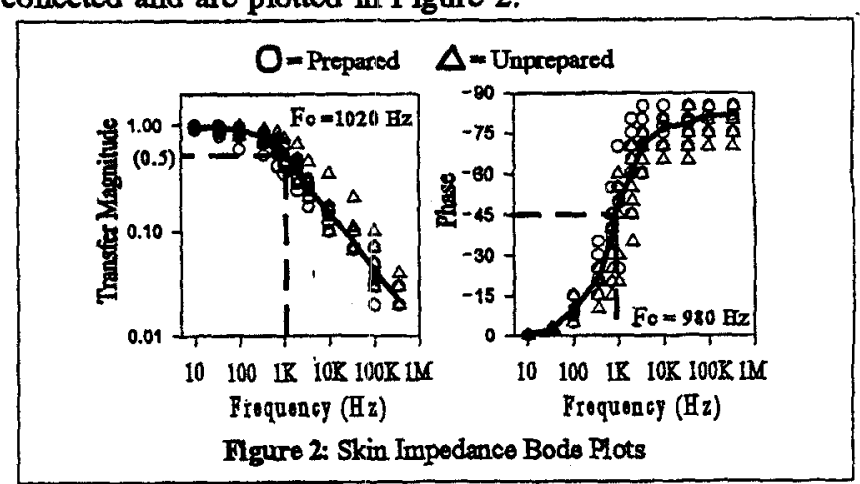

These plots depict the skin's general response as that of a low-pass filter. It is noteworthy that the phase-indicated and magnitude-indicated corner frequencies correspond to each other. Further, these corner frequencies only vary minutely between prepared and unprepared subjects. The average 
comer frequency for a first order low-pass approximation over all subjects is about $1.0 \mathrm{KHz}$, virtually independent of skin resistance.

\section{ANALYSIS OF TENS WAVEFORMS}

Present TENS devices stimulate tissue with a pulse train where the waveforms are short square peaks and have adjustable duty cycles and pulse widths. Typical settings to treat chronic pain are a $20-100 \mathrm{~Hz}$ train with $30-200$ microsecond pulse width. Pulse train options include modulation sequences and burst modes. Amplitudes are set as high as $80 \mathrm{~V}$ per pulse peak as needed by the patient to have adequate pain reduction. The pulse shape was designed to mimic dendritic depolarization (pain signal), in such a way as to cancel the actual pain signal. This pulse shape has been accepted by most manufacturers due to convention and simplicity of required driving circuitry, and is shown in Figure 3a [1]. A prototype TENS device was designed and built by the Armstrong Laboratory, Wright-Patterson AFB, OH for a Dayton OH, VA Hospital TENS study. This prototype device applies a different stimulation waveform, shown in Figure $3 b$, which utilizes a longer pulse base time, a rounded peak, and an extended exponentially decaying trailing edge.

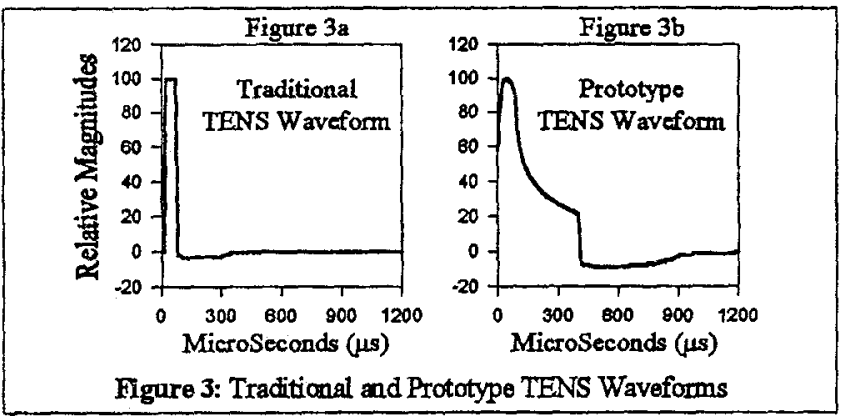

This waveform is driven by very basic circuitry, and would be suitable for production models.

A Fourier analysis of the traditional pulse shows that the output power is evenly distributed throughout lower and mid-range frequencies, as seen in Pigure 4a. The spectral content of the prototype waveform is shown in Figure $4 \mathrm{~b}$. Here, more of the spectral content is at lower frequencies.

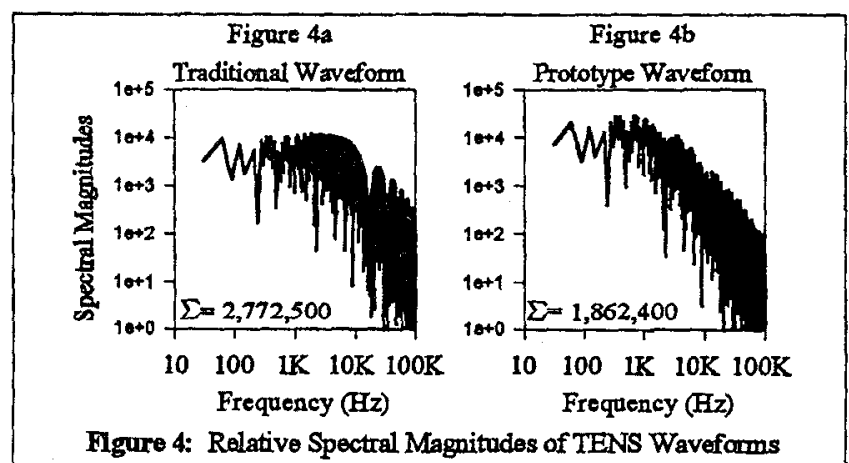

A comparison of spectral power effectiveness can be made on the basis of the ability to transfer real power to the skin. Regardless of the actual mechanism of TENS, as described in gate or endorphin theories [2], in-phase current is necessary for any power transfer. By multiplying the average skin impedance magnitude characteristic and the spectral power distributions of the two waveforms, an effective transfer plot can be made. This plot is shown for a traditional waveform in Pigure 5a and for the prototype waveform in Figure 5b.

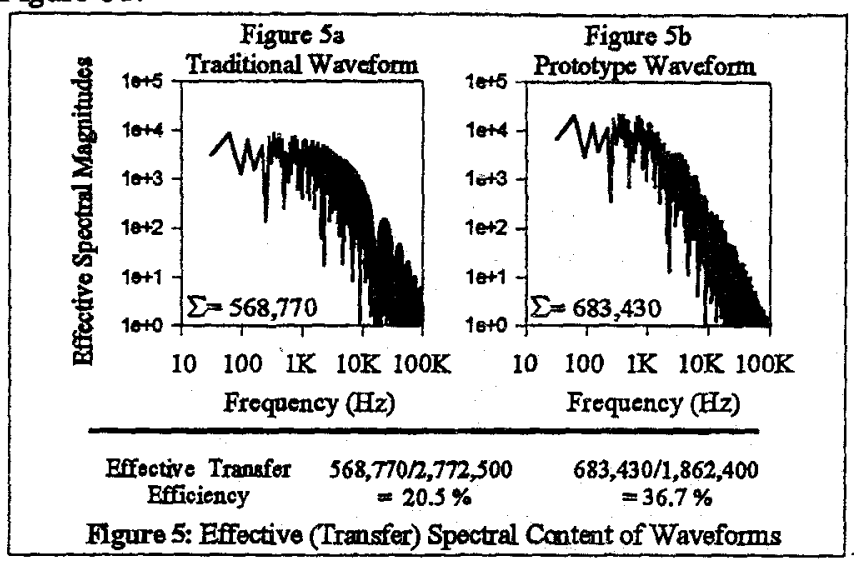

Integrating the effective transfer plots and dividing this sum by the sum of the integral of the entire waveform spectral content results in the effective transfer ratio, which is an indicator of how well the source is spectrally matched to the load. This ratio is calculated for the traditional waveform as $20.5 \%$ and for the prototype waveform as $36.7 \%$. The prototype waveform, thus, has a $80 \%$ greater response efficiency over the traditional TENS waveform. If present TENS derices can be improved by matching the spectral content of the stimulation waveform to the complex impedance of the skin, the stimulation efficiency should be increased. Therefore, peak excitation levels, battery usage, and the requirements of driving components should decrease, improving cost and safety.

Pilot data in experiments with pain sufferers done at the VA Hospital with the prototype unit verified that less electrical output was required for the same level of pain alleviation. Experiments also showed that the waveform was more comfortable than traditional TENS stimulation [3].

\section{CONCLUSIONS}

A modified stimulation waveform that is spectrally matched to skin impedance characteristics with increased efficiency and improved usefulness for TENS devices is presented. It was observed that post-excitation skin may have resonant charge causing errors in resistance measurement. It was found that skin impedance exhibits first order low pass filter characteristics with a $1 \mathrm{KHz}$ corner frequency.

\section{REFERENCES}

[1] "Specifications for Tenzcare No. 6880 Series Stimulator", 3M Health Care, Medical Device Division, 3M Center Bldg. 225-5s-01, May 1989 Specification Sheet.

[2] Raj, P. Prithvi Practical Management of Pain, St Louis: Mosby Year Book, 1992, pp. 1025-1037.

[3] Repperger, D.W., Phillips, C.A., Ho, C.C., and Johnson, D.C.: Phase II VA Hospital Improved TENS Proposal, Pending Submission, 1995. 